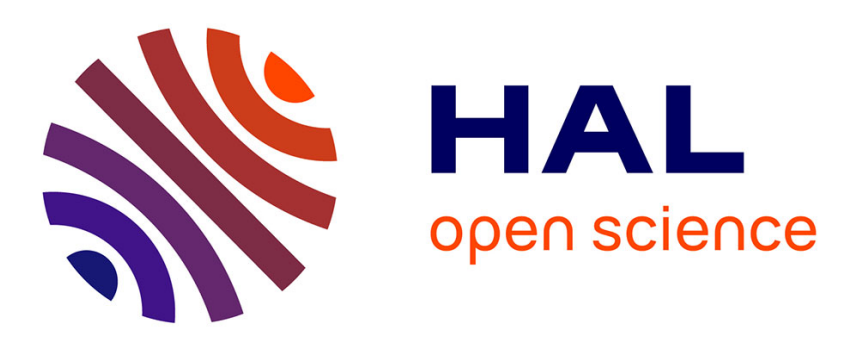

\title{
Appétibilité et caractéristiques organoleptiques des graminées. I. - Comparaison du ray-grass et de la fétuque élevée
}

\author{
Jan Scehovic, Charles Poisson, Michel Gillet
}

\section{To cite this version:}

Jan Scehovic, Charles Poisson, Michel Gillet. Appétibilité et caractéristiques organoleptiques des graminées. I. - Comparaison du ray-grass et de la fétuque élevée. Agronomie, 1985, 5 (4), pp.347-354. hal-00884768

\section{HAL Id: hal-00884768 \\ https://hal.science/hal-00884768}

Submitted on 1 Jan 1985

HAL is a multi-disciplinary open access archive for the deposit and dissemination of scientific research documents, whether they are published or not. The documents may come from teaching and research institutions in France or abroad, or from public or private research centers.
L'archive ouverte pluridisciplinaire HAL, est destinée au dépôt et à la diffusion de documents scientifiques de niveau recherche, publiés ou non, émanant des établissements d'enseignement et de recherche français ou étrangers, des laboratoires publics ou privés. 


\title{
Appétibilité et caractéristiques organoleptiques des graminées. I. - Comparaison du ray-grass et de la fétuque élevée
}

\author{
Jan SCEHOVIC, Charles POISSON (*) \& Michel GILLET (*) \\ Station Fédérale de Recherches Agronomiques de Changins, $\mathrm{CH} 1260 \mathrm{Nyon}$ \\ (*) INRA, Station d'Amélioration des Plantes Fourragères, F 86600 Lusignan
}

Les études du comportement alimentaire des moutons envers différentes espèces de graminées dans une cafétéria d'auges installée à Lusignan (I.N.R.A.-France) ont permis d'enregistrer, entre autres, l'observation suivante : l'aspersion de la fétuque élevée, espèce peu appétible, avec le jus extrait de ray-grass d'Italie, espèce très appétible, et vice-versa, a eu pour résultat la modification significative du comportement des moutons envers les deux espèces comparées. Etant donné que l'effet de ces interventions s'avérait limité dans le temps, nous en avons déduit la présence de substances volatiles.

Afin d'expliquer cette action sur le comportement alimentaire des moutons, nous avons procédé à la détermination de différentes substances chimiques du jus susceptibles d'influencer les caractéristiques organoleptiques de la matière végétale.

Dans le jus extrait par pression de différentes espèces végétales (fétuque élevée, ray-grass anglais, dactyle, raygrass d'Italie, trètle violet et luzerne), nous avons effectué les déterminations suivantes : $\mathrm{pH}$, fraction solide, quelques acides organiques volatils et non volatils, azote total, bases volatiles, $\mathrm{N}\left(\mathrm{NH}_{3}\right)$, phénols totaux et volatils, glucides solubles, substances à carbonyle volatil, esters volatils et composés soufrés. Les résultats de ces analyses nous fournissent les informations suivantes :

- La concentration de la plupart des groupes de substances est supérieure à la $1^{\text {re }}$ coupe par rapport aux coupes suivantes.

- Les différences dans la composition chimique entre le ray-grass et la fétuque élevée, importantes en $1^{\text {re }}$ coupe, s'atténuent au cours de l'année.

- Chez des ray-grass d'Italie plus ou moins sensibles à l'attaque de la rouille couronnée, la concentration des diverses substances varie avec l'importance de l'attaque : dans le même sens pour les substances estimées plutôt dissuasives (bases volatiles, $\mathrm{N}\left(\mathrm{NH}_{3}\right)$ et composés soufrés), en sens inverse pour les substances supposées attractives (aldéhydes et cétones).

- Les différences dans la composition chimique du jus des graminées et des légumineuses sont très importantes.

- Les composés soufrés, abondants dans la fétuque élevée, pourraient être le facteur déterminant de la faible appétibilité de cette espèce.

- Les substances supposées plutôt attractives (esters volatils, aldéhydes, cétones, phénols volatils) pourraient atténuer l'action des substances dissuasives.

- Les matières azotées et les glucides solubles ne semblent pas avoir d'influence sur l'appétibilité des espèces analysées.

Mots clés additionnels : Substances volatiles, pouvoir odorant, jus d'herbe.

Palatibility and organoleptic characteristics of grasses. I. - Comparison between rye-grass and tall fescue.

Studies of alimentary behaviour of sheep towards different species of grasses in a trough cafeteria installed at Lusignan (I.N.R.A., France) allowed us to make, the following observation : spraying tall fescue, a poorly palatable species, with ryegrass juice, and vice-versa, resulted in a significant modification of the behaviour of sheep towards these two species. Since the effect of these treatments was limited in time, we concluded that it was due to volatile substances. In order to explain this action on the alimentary behaviour of sheep, we determined several chemical compounds of the juice likely to influence the organoleptic characteristics of the plant material. In the pressure-extracted juice of different species (tall fescue, perennial ryegrass, cocksfoot, Italian ryegrass, red clover and alfalfa) we determined the following parameters : $\mathrm{pH}$, solid fraction, some volatile and non-volatile organic acids, total nitrogen, volatile bases, ammonia nitrogen, total and volatile phenols, soluble sugars, substances containing volatile carbonyl, volatile esters and sulphur-containing compounds. The results of these analyses gave us the following information :

- the concentration of most groups of substances was higher at the first cut than at subsequent ones ;

- the difference between the chemical composition of Italian ryegrass and that of tall fescues, was large at the first cut but gradually fell during the year ; 


\begin{abstract}
- in Italian ryegrass more or less susceptible to crown rust, the concentration of substances considered as dissuasives (volatile bases, ammonia nitrogen, sulphur-containing compounds) varied in the same way as infection and in the opposite way to substances considered as attractives (aldehydes and ketones) ;

- there were large differences in chemical composition between the juices of grasses and those of legumes ; - the sulphur-containing compounds, abundant in tall fescue, could be the determining factor of the low palatability of this species ;

- the substances considered as attractive (volatile esters, aldehydes, ketones, volatile phenols) could attenuate the effect of dissuasive substances ;

- total nitrogen and soluble sugars did not seem to have any influence on the palatability of the species analysed.
\end{abstract}

Additional key words : Volatile substances, odour intensity, grass juice compounds.

\section{INTRODUCTION}

Les ruminants, comme tous les êtres vivants, vivent dans un environnement extraordinairement complexe auquel ils sont liés par un système de communication chimique. Leur comportement social, reproductif et alimentaire, est déterminé entre autres par des stimuli chimiques. La stimulation, captée par les animaux à l'aide de leurs chemorécepteurs, est traduite par des réactions qui dépendent de l'espèce, de l'individu et de la concentration moléculaire du stimulus chimique.

Le comportement alimentaire des ruminants est très fortement influencé par la composition chimique des aliments qui leur sont offerts. La discrimination alimentaire est bien connue au niveau des conséquences mais relativement peu au niveau de ses causes (UlyatT, 1973).

Il existe une bibliographie abondante qui propose différentes théories sur la sélectivité alimentaire des herbivores et sur les facteurs qui la déterminent : valeur nutritive (DAVIES, 1925 ; TRIBE, 1950 ; COOK \& HARRIS, 1950 ; COOK et al., 1956 ; COWLISHAW \& ALDER, 1960 ; ARNOLD, 1960), composition minérale (FAGAN \& WATKINS, 1932 ; MILTON, 1943 ; STATEN, 1949 ; COOK et al., 1956 ; DAVIES et al., 1959), teneur en azote (HARDISON et al., 1954 ; WEIR \& TORELL, 1959), teneur en glucides solubles et membranaires (Plice, 1952 ; HARdison et al., 1954 ; WeIr \& TORELL, 1959) et relations de ces facteurs avec la sélectivité.

Parmi les premières études faites pour établir des relations entre quelques caractéristiques spécifiques (couleur, odeur, goût) et l'appétence des herbages, on peut citer celles de Tribe (1946), TRIBE \& GORDON (1946) et BeLl (1959). Ces travaux ont mis en évidence l'importance de ces caractéristiques pour la stimulation initiale de l'appétit. Depuis, un intérêt particulier est porté sur le rôle des sens pour la détection de métabolites secondaires des végétaux et sur les effets attractifs ou dissuasifs de ces derniers en relation avec l'appétibilité des herbages (ARNOLD, 1964, 1965, 1970 ; Hugues et al., 1964 ; Mc ClyMONT, 1967 ; ROSENTHAL \& JANZEN, 1979).

En 1972, ARNOLD \& HILL publient les résultats d'un travail très complexe. Leurs observations portent sur l'importance des différents sens dans la sélectivité alimentaire, sur leur interaction et sur les seuils de détection et de rejet de diverses substances chimiques par les ruminants. Les différences individuelles et interspécifiques de comportement alimentaire et les divers mécanismes qui influencent l'ingestibilité et l'appétibilité ont été également étudiés.

La seule faiblesse de leur travail, relevée d'ailleurs par eux-mêmes, est l'utilisation, pour leurs observations, de substances isolées, choisies plus ou moins arbitrairement. Dans leurs conclusions, ils suggèrent une approche plus sûre pour identifier les causes de la faible appétence : il conviendrait d'isoler et d'identifier les substances chimiques contenues dans les espèces ou variétés peu appétibles. Une fois les substances identifiées, les facteurs qui influencent leur concentration peuvent être définis et leur contrôle génétique exploré.

La production et la synthèse de métabolites secondaires coûtent trop cher en énergie pour l'organisme végétal (ROSENTHAL \& JANZEN, 1979). Cela explique probablement le fait que les espèces fourragères, sélectionnées pour le rendement élevé, sont moins riches en métabolites secondaires, donc moins aromatiques que les espèces adventices. Malgré cela, l'incidence des caractéristiques organoleptiques des fourrages sur la sélectivité alimentaire des animaux est évidente, du moins quand ils ont le choix.

Les caractéristiques organoleptiques des fourrages ont leur importance maximum lorsque les animaux ont le choix. Une telle situation, lorsqu'elle est expérimentale, s'appelle une "cafétéria ». GILlET et al. (1983) ont mis au point une cafétéria d'auges dans laquelle on offre à des moutons des quantités égales de différents fourrages fraîchement coupés.

En utilisant cette technique, l'importance de l'odorat dans la différence d'appétibilité entre ray-grass d'Italic et fétuque élevée a été mise en évidence à la Station d'Amélioration des Plantes fourragères de I'I.N.R.A. (Lusignan, France) par aspersion de chaque espèce avec du jus de l'autre (cf. ci-dessous).

Partant de ce résultat, la Station Fédérale de Recherches Agronomiques de Changins (Suisse) a recherché, sur diverses espèces cultivées côte à côte, les éventuelles différences de teneur en substances pouvant être odorantes (Expérience I). Sachant que les attaques de rouilles sur graminées nuisent considérablement à leur appétibilité, cette même Station a étudié l'effet d'une telle attaque sur la teneur de la plante en ces substances odorantes (Expérience II).

Il est évident qu'une substance volatile, ou qu'un groupe de substances volatiles, même présentes en abondance, ne déterminent pas le vrai arôme de la 
matière végétale (NICHOLAS, 1973). L'arôme est le résultat d'un mélange extrêmement complexe de ces substances. Son action attractive ou dissuasive est déterminée par la concentration des substances volatiles, leur proportion, leur volatilité et leur pouvoir odorant.

\section{MISE EN ÉVIDENCE DU PHÉNOMÈNE}

\section{A. Matériel et méthodes}

Le 20 avril 1982, une cafétéria d'auges a permis de comparer à Lusignan, avec 10 moutons, l'appétibilité de 4 fourrages répétés 10 fois. Ces fourrages étaient :

- un ray-grass d'Italie «Adret», semé au printemps 1981,

- une fétuque élevée "Clarine », semée au printemps 1979 sur une parcelle contiguë au ray-grass d'Italie,

- le même ray-grass aspergé avec du jus de la fétuque,

- la fétuque aspergée avec du jus de ray-grass.

Ces jus avaient été extraits immédiatement avant l'expérience, par broyage d'herbe verte et pressage à 80-100 bars. L'aspersion avait été faite avec un petit pulvérisateur à vitres en matière plastique. Les animaux ont été laissés en présence des fourrages pendant $1 \mathrm{~h} 30$ et observés en permanence pendant ce temps.

\section{B. Résultats et conclusion}

Sur $500 \mathrm{~g}$ d'herbe fraîche disponibles par auge, les animaux en ont consommé en moyenne $382 \mathrm{~g}$ pour le ray-grass pur, $186 \mathrm{~g}$ pour le ray-grass aspergé de jus de fétuque, $33 \mathrm{~g}$ pour la fétuque arrosée de ray-grass et $17 \mathrm{~g}$ pour la fétuque pure. Les différences étaient très hautement significatives, entre tous les fourrages, sauf entre les deux formes de fétuque.

Ces résultats semblent montrer que le jus de fétuque serait répulsif, tandis que le jus de ray-grass ne serait pas particulièrement attractif.

Il n'est pas toujours facile quand on observe un animal qui s'approche d'une auge, ou passe devant, puis s'éloigne, de savoir s'il l'a fait par hasard ou s'il avait eu l'intention de manger mais en a été dissuadé par l'odeur. De même, s'il plonge la tête dans l'auge et la retire aussitôt, il n'est pas toujours possible de savoir s'il a goûté ou non au fourrage. Néanmoins, on a pu retirer de cette observation des impressions qui éclairent sans doute les résultats :

- les animaux, au début de l'expérience, ne goûtaient pratiquement pas à la fétuque, ni au ray-grass arrosé de fétuque. Ils mangeaient volontiers le ray. grass pur et semblaient goûter à la fétuque arrosée de ray-grass mais n'insistaient pas ;

- au bout de quelque temps, les animaux se remirent à manger du ray-grass arrosé de jus de fétuque, ce qui explique la consommation relativement bonne de ce dernier.
Tout ceci s'accorderait avec l'hypothèse de l'existence, chez la fétuque, de substances répulsives qui seraient volatiles, ou labiles par oxydation, actions enzymatiques ou autres réactions biochimiques.

Il était donc intéressant de rechercher si les 2 espèces comparées ici différaient par leur teneur en substances odorantes.

\section{RECHERCHE DES SUBSTANCES RESPONSABLES}

\section{A. Substances recherchées : leur intérêt}

Nous avons monté une expérience destinée à permettre différentes analyses chimiques qui pouvaient s'avérer intéressantes. Nous avons choisi de déterminer des groupes de substances et leurs concentrations sans isoler les substances individuelles au sein de chaque groupe. Cela nous permet d'éliminer pour la suite de notre recherche, des groupes de substances sans intérêt apparent.

Le $p H$ du jus d'herbe renseigne sur la forme chimique (acides, bases, sels) de certaines substances du jus. Son importance se situe au niveau de l'action enzymatique.

La fraction solide comprend l'ensemble des substances qui ne se volatilisent pas dans des conditions physiques définies.

L'acidité volatile est la somme des acides volatils (acide acétique, butyrique, propionique, formique, valérique, etc.) présents dans le jus. Le pouvoir odorant de ces substances est très important.

Les acides acétiques et butyrique sont des acides volatils possédant un très fort pouvoir odorant. Leur influence sur l'appétibilité et l'ingestibilité a été étudiée par ARNOLD \& HILL (1972).

Les acides organiques non volatils solubles dans l'eau sont des substances organiques dont la présence peut influencer les qualités gustatives de la matière végétale et l'appétibilité des fourrages (ARNOLD \& Hill, 1972). Certains de ces acides, par exemple tartrique, lactique, citrique, malonique, oxalique, shikimique, quinique, jouent un rôle prépondérant pour la détermination du $\mathrm{pH}$ du suc vacuolaire. Ils représentent le plus souvent des produits intermédiaires du métabolisme des glucides, des lipides ou des acides aminés.

L'acide tartrique a suscité notre intérêt à cause de sa plus forte acidité que les autres acides organiques et à cause de son action positive sur la qualité gustative dans l'alimentation humaine.

$L$ 'azote total est souvent considéré comme un facteur déterminant de l'appétibilité des fourrages. Son abondance dans les plantes jeunes et dans les parties plus appétibles (feuilles) donne l'impression d'être la cause de la préférence des animaux pour ces dernières.

L'azote ammoniacal est souvent considéré comme répulsif.

Les bases volatiles sont représentées essentiellement dans la matière végétale par les amines volatiles et l'ammoniac. Ces substances azotées ont un pouvoir odorant très prononcé et leur action sur les animaux peut être plutôt dissuasive. 
Les glucides solubles sont un groupe de substances organiques englobant un nombre important de différents sucres et leurs dérivés. Leur composition en glucides individuels, dont l'intensité édulcorante est très variée, dépend de l'espèce végétale. Les résultats de l'analyse varient aussi selon la méthode d'extraction. En outre, on a pu observer des variations très importantes dans les réactions de différentes espèces animales par rapport à des solutions "sucrées " (GOATCHER \& CHURCH, 1970a, $b$ ).

Les composés phénoliques sont des substances aromatiques du métabolisme secondaire plus ou moins solubles dans l'eau. C'est un groupe de substances extrêmement vaste de caractère et de fonction très divers. Leur activité physiologique au sein de l'organisme végétal est très importante. On leur attribue une fonction écologique certaine à travers leurs effets sur la physiologie des animaux (RoBINSON, 1980). En effet, certains phénols dans la matière végétale ont une action dissuasive envers les herbivores : ils peuvent provoquer des irritations après leur ingestion et peuvent même être toxiques. D'autres ont une action attractive pour des pollinisateurs et également pour les herbivores par leur odeur et par leur capacité d'absorber la lumière ultraviolette.

Les aldéhydes et les cétones sont des substances organiques caractérisées par leur groupement fonctionnel - groupement carbonyle. Malgré leur faible concentration dans les plantes, ils présentent un intérêt particulier par leur contribution à la formation des parfums spécifiques de matériaux d'origine végétale. Le 2-héxénal qu'on appelle aussi « aldéhyde de feuilles " est responsable de fortes odeurs distinctives de feuilles broyées. Il n'y a pas très longtemps encore, l'odeur agréable de beaucoup de plantes était attribuée aux aldéhydes; cela est contesté aujourd'hui (NichOLAS, 1973 ; ROBINSON, 1980).

Les esters volatils contribuent à la formation des parfums des plantes. Leur action sur les herbivores serait plutôt attractive.

Les composés soufrés sont des substances volatiles caractérisées par une odeur très désagréable. Ils peuvent être détectés par l'odorat à des concentrations extrêmement faibles ( 1 partie pour 50 milliards). Biologiquement, ils sont dérivés probablement des acides aminés. En outre, les composés soufrés peuvent être un artefact résultant de la réaction de certaines substances avec du soufre. On les trouve dans le matériel biologique sous forme de thiols (mercaptans) et de sulfides. L'oignon et l'ail sont les meilleurs exemples de la participation des composés soufrés à la formation de l'arôme des plantes (ROBINSON, 1980).

Les thiols se transforment facilement en disulfides par oxydation à l'air. Ces derniers sont moins volatils mais leur odeur est plus agressive. Cela peut avoir des conséquences dans les fourrages conservés.

\section{B. Matériel et méthodes}

\section{Description de l'essai (tabl. 1)}

L'essai a été conduit à la Station de Changins, grâce à l'aimable collaboration de S. BADOUX. Il a permis d'élargir la comparaison à d'autres espèces que le ray-grass et la fétuque élevée, sachant que cette dernière est connue pour avoir la moins bonne appétibilité.

Les graminées étudiées (Expérience I) ont été semées en 4 répétitions au printemps 1981, en lignes de $3 \mathrm{~m}$ de longueur, écartées de $50 \mathrm{~cm}$, avec fertilisation $\mathrm{N}, \mathrm{P}, \mathrm{K}$ respectivement de $250,130,100 \mathrm{~kg} / \mathrm{ha}$. Elles ont été exploitées en 5 coupes.

Les ray-grass d'Italie (Expérience II) de même que les 2 légumineuses analysées à titre de comparaison ont été semés à la même époque que les 3 graminées de l'expérience I, mais dans les parcelles expérimentales $\left(10,5 \mathrm{~m}^{2}\right)$. Nous avons choisi pour les graminées la $3^{\text {e }}$ coupe à cause des différences bien marquées de l'infection par la rouille couronnée. Le tableau 1 récapitule les dates de coupes soumises à l'analyse.

\section{Procédés utilisés pour les prélèvements et les analyses chimiques}

Pour analyser des substances odorantes, donc volatiles, il est indispensable d'opérer sur de l'herbe frâ̂che.

La matière végétale (tabl. 1) destinée à être analysée a été récoltée toujours à la même heure de la journée. 5 à $10 \mathrm{~kg}$ de cette matière ont été immédiatement hachés et soumis à une pression de 150 bars environ dans un pressoir hydraulique.

TABLEAU

Espèces et variétés mises à l'essai. Dates de prélèvement de la matière végétale pour analyse. Tested species and varieties. Dates of sampling of plant material for analysis.

\begin{tabular}{|c|c|c|c|c|c|c|c|}
\hline \multirow{2}{*}{\multicolumn{2}{|c|}{ Espèces }} & \multirow{2}{*}{ Variété } & \multicolumn{5}{|c|}{ Coupes } \\
\hline & & & $1 *$ & $2^{* *}$ & 3 & 4 & 5 \\
\hline \multirow{3}{*}{ 宓 } & Ray-grass anglais & Melino & $29-4$ & $22-6$ & $22-7$ & $26-8$ & $29-9$ \\
\hline & Fétuque élevée & Barcel & $29-4$ & $22-6$ & $22-7$ & $26-8$ & $29-9$ \\
\hline & Dactyle & Neo-Lara & & $22-6$ & $22-7$ & $26-8$ & $29-9$ \\
\hline \multirow{5}{*}{ 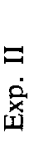 } & Luzerne & Vertus & & $8-6$ & & & \\
\hline & Trèfle violet & Renova & & $8-6$ & & & \\
\hline & Ray-grass Italie & Bar DK 81-115 & & & $29-9$ & & \\
\hline & & Bar DK 81-106 & & & $29-9$ & & \\
\hline & & G 764 & & & $29-9$ & & \\
\hline
\end{tabular}

* Stade « Epi à $10 \mathrm{~cm} » ; * *$ Remontaison. 
Le jus recueilli dans plusieurs petits récipients $(50 \mathrm{ml})$ en plastique a été immédiatement fixé dans l'azote liquide. Pour éviter la dénaturation et la modification de la composition chimique du jus, dues à l'action des enzymes et à d'autres réactions biochimiques du jus, les manipulations entre la récolte et la fixation et surtout entre l'extraction par pression et la stabilisation doivent être extrêmement rapides (20-30 mn). La distribution du jus et son stockage dans le congélateur en petites portions (1 prise d'analyse environ) permet d'éviter la recongélation après prélèvement du jus pour analyse.

L'isolation et le dosage des substances volatiles sont des procédés très délicats, non seulement à cause de la concentration très faible mais aussi en raison des risques de pertes en cours de manipulation. Une multitude de précautions au niveau de la conservation, de l'isolation et de l'analyse sont indispensables. Nous avons mis au point nos propres techniques d'analyse, à partir de nos équipements, de la nature de la matière à analyser. etc. Le but principal de notre étude est de « déblayer le terrain » dans un domaine très peu connu. Nous avons opté pour la distillation par l'entraînement à la vapeur d'eau et à l'azote comme procédé d'isolation des substances volatiles. L'avantage de cette méthode par rapport à d'autres (distillation sous pression réduite, extraction par des solvants, etc.) est sa rapidité. Elle correspond surtout mieux aux conditions naturelles. En fait, en appliquant des procédés plus «violents », plus exhaustifs, on risque d'extraire des substances faiblement volatiles qui, dans les conditions naturelles, n'atteindraient probablement pas les chémorécepteurs des animaux.

Les résultats sont des valeurs obtenues dans des conditions analytiques strictement standardisées, donc reproductibles, mais pas forcément absolues. Pour illustrer ce fait, on peut mentionner le cas des acides gras volatils. Ils sont très volatils jusqu'à l'acide caproïque $\left(\mathrm{C}_{6}\right)$. Les acides caprilique $\left(\mathrm{C}_{8}\right)$ et caprique $\left(C_{10}\right)$ sont faiblement volatils et il faut une très longue distillation ou l'extraction par des solvants organiques pour les isoler complètement.

Le $\mathrm{pH}$ du jus d'herbe a été déterminé à l'aide du pHmètre étalonné avec des solutions standard.

La fraction solide a été déterminée par évaporation de $10 \mathrm{ml}$ de jus à basse température $\left(70^{\circ}\right)$ jusqu'à stabilisation du poids.

L'extraction des acides volatils a été effectuée à l'aide de vapeur d'eau produite par un générateur. La vapeur entraîne les acides volatils lors de son passage dans le jus $(25 \mathrm{ml})$. Après condensation dans un réfrigérant, elle est captée dans un récipient placé dans un bain de glace. Le distillat est titré par $\mathrm{NaOH} 0,1 \mathrm{~N}$ en présence de phénol-phtaléine. Il est indispensable avant de commencer la distillation de s'assurer que l'eau dans le générateur de vapeur est exempte de $\mathrm{CO}_{2}$ (acidité de la vapeur). L'acidité volatile du jus, exprimée en $\mathrm{g} / \mathrm{l}$ d'acide acétique, s'obtient en multipliant par 0,24 (pour les $25 \mathrm{ml}$ de jus) le nombre de $\mathrm{ml} \mathrm{de}$ $\mathrm{NaOH} 0,1 \mathrm{~N}$ utilisé pour la titration.

Les acides acétique et butyrique ont été dosés par distillation fractionnée et titration du distillat par $\mathrm{NaOH} 0,1 \mathrm{~N}$. Les résultats ont été obtenus à partir du nombre de $\mathrm{ml}$ de $\mathrm{NaOH} 0,1 \mathrm{~N}$ utilisé pour la titration, à l'aide d'équations établies préalablement avec des quantités connues d'acides gras volatils. A titre de comparaison, nous avons calculé les « autres acides volatils » à l'aide d'une équation établie empiriquement :

autres ac. volat. = acidité volatile -

$$
\text { - (acide acétique + ac. butyrique) . }
$$

Les acides organiques non volatils, transformables par oxydation en acides volatils, ont été dosés d'après le même procédé que ces derniers.

Utilisant la propriété de l'acide tartrique de former un complexe coloré en jaune-rose avec le vanadate d'ammonium, nous l'avons dosé colorimétriquement, à partir d'une courbe d'étalonnage.

L'azote total a été dosé selon la méthode classique de Kjeldahl.

L'azote ammoniacal a été dosé selon une méthode inspirée de CONWAY (1947), basée sur l'absorption par l'acide borique à 1 p. 100 additionné d'un indicateur (bromocrésol + rouge de méthyle), de l'ammoniac diffusé à partir de jus d'herbe. La quantité d'azote ammoniacal est déterminée par titration en retour avec $\mathrm{HCl}, 0,01 \mathrm{~N}$. Le nombre de $\mathrm{ml}$ de l'acide utilisé pour cette titration par rapport à celui utilisé pour la titration d'une solution avec une concentration en $\mathrm{NH}_{3}$ connue, sert de base de calcul de la concentration de $\mathrm{NH}_{3}$ dans le jus d'herbe.

Les bases volatiles, faciles à extraire du jus d'herbe fortement basifié (par distillation par l'entraînement à la vapeur), ont été captées dans l'acide borique. Leur quantité a été calculée à partir du nombre de $\mathrm{ml}$ d'acide sulfurique $0,1 \mathrm{~N}$ utilisé pour la neutralisation de bases dans le distillat. Nous n'avons pas pris la précaution de désactiver les amines oxydases, donc les résultats n'ont qu'une valeur comparative.

Les glucides solubles se trouvent dans le jus d'herbe sous forme de mono- et oligosaccharides, et de polysaccharides solubles dans l'eau. Ils ont été déterminés par colorimétrie grâce à leur propriété de produire un complexe jaune-orange avec le phénol dans un milieu fortement acide.

Les composés phénoliques, d'une façon générale, ne sont pas volatils et ne peuvent donc pas être extraits par l'entraînement à la vapeur d'eau. Par contre, des esters et des éthers phénoliques, moins polaires, sont volatils et participent souvent à la composition des essences végétales. La capacité des composés phénoliques de réduire, en milieu alcalin, le complexe formé par les réactifs phosphotungstique et molybdique, a été utilisée pour leur dosage colorimétrique. Les phénols solubles présents dans le jus d'herbe et ceux extraits par distillation par entraînement à la vapeur d'eau (éthers et esters phénoliques volatils), mélangés au réactif spécifique et à $\mathrm{Na}_{2} \mathrm{CO}_{3}$ à $20 \mathrm{p}$. 100 , donnent une coloration bleue (oxydes de tungstène et de molybdène) dont l'intensité est mesurée à l'aide d'un spectrophotomètre. Les résultats sont calculés à l'aide d'une courbe d'étalonnage établie avec de l'acide gallique.

Les aldéhydes et les cétones, substances à carbonyle volatil, sont facilement extractibles par distillation par l'entraînement à la vapeur d'eau. Ils ont été extraits du jus d'herbe par distillation dans un milieu tamponné $(\mathrm{pH}=9)$. La réaction entre les substances à carbonyle volatil présentes dans le distillat et la 2,4-dinitrophénylhydrazine dans le milieu basique a 
pour résultat une coloration rouge dont l'intensité est mesurée dans un spectrophotomètre. La réaction dure 15 à $18 \mathrm{~h}$. La concentration des substances à carbonyle volatil a été calculée à l'aide d'une courbe d'étalonnage établie avec aldéhyde acétique.

Les esters volatils ont été extraits du jus d'herbe par distillation par entraînement à la vapeur d'eau. Les esters présents dans le distillat sont saponifiés à chaud par NaOH $0,1 \mathrm{~N}$, ajouté en excès. La quantité de base utilisée pour la saponification est déterminée par la titration avec $\mathrm{H}_{2} \mathrm{SO}_{4} 0,1 \mathrm{~N}$. Le nombre de $\mathrm{ml}$ de $\mathrm{NaOH} 0,1 \mathrm{~N}$ utilisé pour la saponification des esters, multiplié par 8,8 , donne la quantité des esters volatils exprimée en $\mathrm{mg}$ d'acétate d'éthyle.

Les composés soufrés volatils ont été libérés par action de l'acide sulfurique. Ils ont été ensuite entraînés par un courant d'hélium ou d'azote extra pur, puis absorbés sur de l'hydroxyde de cadmium fraîchement préparé. L'addition de $\mathrm{N}, \mathrm{N}$ diméthyl-p-phénylènediamine, dissous dans $\mathrm{H}_{2} \mathrm{SO}_{4}$ en présence de $\mathrm{Fe}^{+++}$, provoque une réaction qui a pour résultat la formation de bleu de méthylène qui est mesuré par spectrophotométrie.

Les résultats ont été calculés à l'aide d'une courbe d'étalonnage établie préalablement avec $\mathrm{Na}_{2} \mathrm{~S}$. Chaque détermination a été accompagnée d'un témoin qui permettait de vérifier la pureté des résultats.

\section{Résultats}

\section{Expérience I (tabl. 2)}

Les concentrations en divers groupes de substances dans le jus de la $1^{\text {re }}$ coupe sont relativement élevées. De même, les différences entre les 2 espèces de graminées sont bien marquées. La situation change complètement pour les autres coupes. On enregistre une diminution de concentration très importante et les différences entre les espèces végétales étudiées s'atténuent sans régularité apparente.

En considérant les différences dans la composition chimique entre les espèces végétales et la cohérence de leur évolution tout au long de la période de végétation, nous observons des valeurs régulières chez 3 groupes de substances volatiles : substances à carbonyle volatil, esters volatils et composés soufrés. Les autres substances ou groupes, y compris ceux souvent cités (matière azotée, glucides ou acides organiques)

TABLEAU 2

Expérience I : Composition chimique du jus de trois graminées lors de 5 coupes successives $(R g=R a y$-grass anglais, $F e=$ Fétuque élevée $; D g=$ Dactyle $)$.

Experiment I: Chemical composition of juice of three grasses at 5 successive cuts $(R g=$ ryegrass $; F e=$ tall fescue; Dg $=$ cocksfoot $)$.

\begin{tabular}{|c|c|c|c|c|c|c|c|c|c|c|c|c|c|c|}
\hline Coupes & 1 & & & 2 & & & 3 & & & 4 & & & 5 & \\
\hline Déterminations & $\operatorname{Rg}$ & $\mathrm{Fe}$ & $\mathrm{Rg}$ & $\mathrm{Fe}$ & $\mathrm{Dg}$ & $\mathrm{Rg}$ & $\mathrm{Fe}$ & $\mathrm{Dg}$ & $\mathrm{Rg}$ & $\mathrm{Fe}$ & $\mathrm{Dg}$ & $\operatorname{Rg}$ & $\mathrm{Fe}$ & Dg \\
\hline $\mathrm{pH}$ & 5,83 & 6,14 & 5,11 & 5,76 & 6,07 & 5,69 & 5,78 & 5,76 & 5,81 & 5,81 & 5,76 & 5,86 & 5,83 & 5.80 \\
\hline Fraction solide & 104,45 & 59,85 & 29,39 & 31,91 & 22,15 & 45,72 & 45,75 & 27,24 & 48,74 & 50,24 & 37,02 & 47,30 & 41,54 & 32,24 \\
\hline Acidité volatile (expr. en ac. acétique) & - & - & 0,294 & 0,245 & 0,257 & 0,324 & 0,266 & 0,238 & 0,481 & 0,331 & 0,310 & 0,314 & 0,317 & 0,209 \\
\hline Acide acétique & 0,589 & 0,355 & 0,243 & 0,183 & 0,189 & 0,301 & 0,219 & 0,200 & 0,452 & 0,157 & 0,138 & 0,256 & 0,192 & 0,189 \\
\hline Autres acides volat. & - & - & 51 & 50 & 68 & 23 & 33 & 38 & 29 & 161 & 145 & 58 & 125 & 80 \\
\hline Acides non volatils solubles dans l'eau g/l & 20,34 & 6,86 & 1,213 & 1,494 & 0,799 & 3,08 & 3,23 & 1,164 & 3,682 & 4,455 & 2,330 & 3,92 & 3,35 & 1,866 \\
\hline Acide tartrique $\quad \mathrm{mg} / 1$ & 338 & 178 & 117 & 0,0 & 0,0 & 245 & 202 & 234 & 214 & 196 & 240 & 187 & 196 & 207 \\
\hline Azote total & 2,541 & 1,912 & 0,993 & 0,973 & 0,615 & 0,928 & 1,421 & 0,505 & 1,129 & 1,184 & 0,587 & 1,442 & 1,189 & 0,690 \\
\hline Bases volatiles & 213 & 169 & 78 & 80 & 48 & 95 & 133 & 50 & 130 & 110 & 66 & 150 & 110 & 64 \\
\hline $\mathrm{N}\left(\mathrm{NH}_{3}\right)$ & 114 & 30 & 0,0 & 2 & 0,0 & 34 & 21 & 10 & 44 & 10 & 12 & 29 & 15 & 14 \\
\hline Phénols totaux & 2,100 & 1,567 & 0,591 & 0,573 & 0,857 & 1,159 & 1,008 & 1,195 & 1,212 & 1,354 & 1,843 & 1,132 & 0,999 & 1,496 \\
\hline Ethers + esters phénol. & 23,5 & - & 14,2 & 12,8 & 8,6 & 18,7 & 17,3 & 10,6 & 14,1 & 15,2 & 12,3 & 12,1 & 11,2 & 9,8 \\
\hline Glucides solubles & 62,72 & 26,14 & 5.62 & 7.41 & 1,76 & 15,73 & 13,95 & 3,84 & 9,19 & 20,20 & 7,11 & 15,43 & 15,14 & 7,41 \\
\hline Composes soutres & 140 & 340 & 0,0 & 30 & 0,0 & 0,0 & 140 & 10 & 4 & 200 & 2 & 0,0 & 128 & 6 \\
\hline
\end{tabular}

\section{TABLEAU 3}

Expérience 1: Concentration des composés soufrés dans le résidu. Experiment I : Concentration of sulphur compounds in the residue.

\begin{tabular}{ccccccccccc}
\hline \hline $\begin{array}{c}\text { Matière } \\
\text { végétale }\end{array}$ & $\begin{array}{c}\text { Trèfle } \\
\text { violet }\end{array}$ & Luzerne & & Fétuque élevée & $\begin{array}{c}\text { Ray-grass } \\
\text { anglais }\end{array}$ \\
\hline $\begin{array}{c}\text { Coupe } \\
\text { Composés } \\
\text { soufrés }\end{array}$ & 3 & 3 & 1 & 2 & 3 & 4 & 5 & 1 \\
$\begin{array}{c}\text { en \% de la } \\
\text { quantité } \\
\text { initiale }\end{array}$ & 3,6 & 19,4 & 51,6 & 0 & 42,1 & 28,3 & 9,6 & 0 \\
\hline
\end{tabular}

ne semblent pas, ou peu, avoir d'influence sur l'appétibilité.

Après avoir enregistré chez la fétuque élevée l'importance quantitative des composés soufrés volatils et connaissant leur très fort pouvoir odorant, nous avons étudié plus en détail leur présence après diverses opérations. Ces observations avaient pour but de vérifier des modifications éventuelles de la concentration en composés soufrés au cours de la conservation de la matière végétale. Nous avons contrôlé leur concentration dans le résidu (fraction solide) après évaporation du jus à basse température (tabl. 3). Les composés soufrés dans le résidu se trouvent probablement sous forme de disulfides. 
Nous avons également dosé les composés soufrés, sans extraction du jus, dans de l'herbe ( $3^{\mathrm{e}}$ groupe) conservée par séchage dans une étuve ou lyophilisée (tabl. 4).

\section{TABLEAU 4}

Expérience I : Concentration des composés soufrés dans de l'herbe conservée.

Experiment I: Concentration of sulphur compounds in stored grass.

\begin{tabular}{lcc}
\hline \hline & \multicolumn{2}{c}{ Mode de conservation : } \\
\hline & $\begin{array}{c}\text { Séchage à l'étuve } \\
\mu \mathrm{g} / \mathrm{g}\end{array}$ & $\begin{array}{c}\text { Lyophilisation } \\
\mu \mathrm{g} / \mathrm{g}\end{array}$ \\
\hline Ray-grass anglais & 59 & 176 \\
Fétuque élevée & 294 & 344 \\
Dactyle & 109 & 160 \\
\hline \hline
\end{tabular}

Les composés soufrés trouvés pourraient être des fractions faiblement volatiles, des thiols oxydés, des artefacts produits en cours d'analyse par décomposition des substances non volatiles ou des acides aminés soufrés produits par l'action de l'acide sulfurique concentré. Le dosage des acides aminés soufrés, précurseurs probables des composés soufrés volatils, n'indique que des différences non significatives entre les 3 espèces de graminées analysées.

\section{Expérience II (tabl. 5)}

Cette expérience a été effectuée parallèlement aux tests en cafétéria (SFRA Changins) avec des moutons qui ont confirmé leur préférence significative pour les variétés de ray-grass d'Italie résistant à l'attaque de la rouille couronnée, Puccinia coronata (SCHMIDT, comm. pers.).

Les relations entre la concentration de divers groupes de substances et la présence de la rouille sont évidentes dans la plupart des cas. Les concentrations en substances considérées comme étant plutôt attractives pour l'animal, évoluent dans le sens inverse de celles dont l'action ne peut être que dissuasive (bases volatiles, $\mathrm{N}\left(\mathrm{NH}_{3}\right)$ et composés soufrés). La crédibilité de cette expérience est légèrement amoindrie par le fait qu'il s'agit de 3 variétés différentes dont on ne connaît pas la composition chimique de départ (sans rouille).

Les analyses du jus de 2 légumineuses (tabl. 3), effectuées à titre de comparaison, montrent des différences parfois très importantes entre les 2 familles de plantes fourragères.

Nous avons procédé pour les 2 expériences à quelques déterminations complémentaires $\left(\mathrm{SO}_{2}, \mathrm{HCN}\right.$, tanins, alcools) qui n'ont apporté aucune information supplémentaire valable.

\section{CONCLUSIONS}

Les substances dont on parle habituellement (azote, glucides solubles) n'ont pas semblé avoir une forte influence sur l'appétibilité des fourrages ; par contre, cette dernière pourrait bien être fortement influencée par l'action résolument dissuasive des composés soufrés volatils. Ceux-ci sont nettement plus concentrés dans la fétuque élevée que dans les autres espèces ; c'est peut-être l'une des raisons de l'infériorité relative de l'appétibilité de cette espèce. L'augmentation de leur teneur en relation avec la contamination par la rouille chez le ray-grass d'Italie et leur concentration

\section{TABLEAU 5}

Expérience II : Composition chimique du jus de trèfle violet (Tr), de luzerne (Lu) et de ray-grass d'Italie plus ou moins sensibles à la rouille couronnée.

Experiment II : Chemical composition of juice of red clover (Tr), alfalfa (Lu) and Italien ryegrass more or less susceptible to crown rust.

\begin{tabular}{|c|c|c|c|c|c|c|}
\hline Déterminations & \multicolumn{2}{|r|}{$\operatorname{Tr}$} & $\mathrm{Lu}$ & \multicolumn{3}{|c|}{ Ray-grass d'Italie* } \\
\hline $\mathrm{pH}$ & & 5,44 & 5,47 & 5,87 & 6,28 & 6,78 \\
\hline Fraction solide & $\mathrm{g} / 1$ & 70,94 & 81,78 & 39,36 & 45,70 & $48,7 \overline{7}$ \\
\hline Acidité volatile (expr. en acide acétique) & $\mathrm{g} / 1$ & 0,623 & 0,763 & 0,368 & 0,362 & 0,495 \\
\hline Acide acétique & $\mathrm{g} / \mathrm{l}$ & 0,619 & 0,684 & 0,222 & 0,235 & 0,326 \\
\hline Acide butyrique & $\mathrm{mg} / 1$ & 0,0 & 0,0 & 0,0 & 0,09 & 31 \\
\hline Autres acides volatils & $\mathrm{mg} / \mathrm{l}$ & 4 & 79 & 146 & 118 & 138 \\
\hline Acides non volatils solubles dans l'eau & $\mathrm{g} / 1$ & 16,96 & 11,49 & 2,589 & 2,972 & 3,799 \\
\hline Acide tartrique & $\mathrm{mg} / \mathrm{l}$ & 220 & 249 & 174 & 205 & 178 \\
\hline Azote total & $\mathrm{g} / 1$ & 1,341 & 2,955 & 0,721 & 0,924 & 1,352 \\
\hline Bases volatiles & $\mathrm{mg} / 1$ & 173 & 390 & 72 & 105 & 166 \\
\hline $\mathrm{N}\left(\mathrm{NH}_{3}\right)$ & $\mathrm{mg} / 1$ & 83 & 289 & 21 & 36 & 79 \\
\hline Phénols totaux & $\mathrm{g} / 1$ & 1,310 & 2,020 & 0,946 & 0,981 & 1,345 \\
\hline Ethers + esters phénol. & $\mathrm{mg} / 1$ & 21,4 & 34,0 & 10,7 & 12,3 & 13,4 \\
\hline Glucides solubles & $\mathrm{g} / \mathrm{l}$ & 24,06 & 19,60 & 15,43 & 20,49 & 13,95 \\
\hline Aldéhydes + cétones (carbonyle volat.) & $\mathrm{mg} / 1$ & 10,111 & 22,821 & 6,631 & 6,212 & 0,0 \\
\hline Esters volatils (expr. en éthyl acétate) & $\mathrm{mg} / 1$ & 77,4 & 77,6 & 105,6 & 112,6 & 213,0 \\
\hline Composés soufrès & $\mathrm{mg} / \mathrm{l}$ & 90 & 175 & 86 & 124 & 137 \\
\hline
\end{tabular}

* $R=$ variété résistante - sans rouille.

$M S=$ variété moyennement sensible -30 à $40 \mathrm{p} .100$ feuilles atteintes.

$S=$ variété sensible -70 à 80 p. 100 feuilles atteintes.

$R=$ resistant cultivar - without rust.

$M S=$ moderately susceptible cultivar -30 to $40 \%$ of leaves affected.

$S=$ susceptible cultivar - 70 to $80 \%$ of leaves affected. 
relativement élevée dans la luzerne par rapport au trèfle violet semblent confirmer l'action négative des composés soufrés sur l'appétibilité de ces fourrages. Par ailleurs, cette action des composés soufrés est peut-être atténuée dans la luzerne et dans le ray-grass anglais ( $1^{\text {re }}$ coupe) par la présence abondante d'autres substances (aldéhydes, cétones, phénols volatils) dont l'action est plutôt attractive.

Cette étude a été réalisée pour comparer l'appétibilité de différentes espèces. Il existe également, par suite de la sélection, une forte variabilité entre génotypes d'une même espèce, la fétuque élevée. Ces différences sont-elles de même nature que les précédentes?
Une nouvelle expérimentation sera réalisée pour le savoir.

Reç le 28 juin 1983. Accepté le 23 novembre 1984.

\section{REMERCIEMENTS}

C'est avec plaisir que nous remercions M. S. BADoux d'avoir mis à notre disposition certains de ses essais de comparaison d'espèces et de variétés.

\section{RÉFÉRENCES BIBLIOGRAPHIQUES}

Arnold G. W., 1960. Selective grazing by sheep of two forage species at different stages of growth. Aust. J. Agric. Res., 11, 1026.

Arnold G. W., 1964. Factors within plant associations affecting the behaviour and performance of grazing animals, p. 133. In Crisp D. T. : "Grazing in terrestrial and marine environments", Blackwell, Oxford.

Arnold G. W., 1965. The special senses in grazing animals. Aust. $J$. Agric. Res., 17, 531.

Arnold G. W., 1970. Regulation of food intake in grazing ruminants, p. 264. In Philipson A. T. : « Physiology of digestion and metabolism in the ruminant $)$.

Arnold G. W., Hill J. J., 1972. Chemical factors affecting selection of food plants by ruminants, p. 72. In Harborne J. : «Phytochemical Ecology 》, Academic Press, New York.

Bell F. R., 1959. Preference thresholds for taste discrimination in goats. J. Agric. Sci., 52, 125.

Conway E. J., 1947. Microdiffusion and volumetric analysis error. Crosby, Lockwood \& Son, London.

Cook C. W., Harris L. E., 1950. The nutritive content of the grazing sheep's diet on summer and winter ranges in Utah. Bull. 342 Utah Agric. Exp. Sta.

Cook C. W., Stoddart L. A., Harris L. E., 1956. Comparative nutritive value and palatability of some introduced and native forage plants. Bull. 385 Utah Agric. Exp. Sta.

Cowlishaw S. J., Alder F. E., 1960. The grazing preference of cattle and sheep. J. Agric. Sci., 54, 257.

Davies W., 1925. The relative palatability of pasture plants. $J$. Minist. Agric. U.K., 32, 106.

Davies R. O., Jones D. T. H., Milton W. E., 1959. Factors influencing the composition and value of herbage from fescue and Molinia areas. J. Agric. Sci., 53, 268.

Fagan T. W., Watkins H. T., 1932. The chemical composition of the miscellaneous herbs of pastures. Welsh J. Agric., 8, 144.

Gillet M., Noel C., Jadas-Hecart J., 1983. La cafétéria d'auges. Méthode d'étude de l'appétibilité des fourrages. Agronomie, 3 (8), 817-822.

Goatcher W. D., Church D. C., 1970a. Taste responses in ruminants. I. Reactions of sheep to sugars, saccharin, ethanol and salts. J. Anim. Sci., 30, 777.
Goatcher W. D., Church D. C., 1970b. Taste responses in ruminants. II. Reactions of sheep to acids, quinine, urea and sodium hydroxide. J. Anim. Sci., 30, 784.

Hardison W. H., Reid J. T., Martin C. M., Woolfolk R. G., 1954. Degree of herbage selection by grazing cattle. J. Dairy Sci., 37, 89.

Hugues R. E., Milher C., Dale J., 1964. Selectivity in grazing, p. 185. In Crisp D. T. " Grazing in terrestrial and marine environments ", Blackwell, Oxford.

McClymont G. L., 1967. Selectivity and intake in the grazing ruminant. Handb. Physiol., 1, 129.

Milton W. E., 1943. The yields of ribwort plantain (ribgrass), when sown in pure plots and with grass and clover species. Welsh $J$. Agric., 17, 109.

Nicholas H. J., 1973. Miscellaneous volatile plant products, p. 381 In Miller : "Phytochemistry vol. II ", Litton Educational Publ. $445 \mathrm{p}$

Plice M. J., 1952. Sugar versus the intuitive choice of foods by livestock. J. Range Mgmt., 5, 69.

Robinson T., 1980. The organic constituents of higher plants (Cordus Press, North Amherst, Mass.), 352 p.

Rosenthal G. A., Jansen D. H., 1979. Herbivores. Their Interaction with Secondary Plant Metabolites. Academic Press, $718 \mathrm{p}$.

Staten H. W., 1949. Palatability trials of winter pasture crops and effects of phosphate fertilisers on palatability. Tech. Bull. T-35, Okla. Agric. Exp. Sta.

Tribe D. E., 1946. The importance of the sense of smell to the grazing sheep. J. Agric. Sci., 19, 309.

Tribe D. E., 1950. The behaviour of the grazing animal. A critical review of present knowledge. J. Br. Grassl. Soc., 5, 209.

Tribe D. E., Gordon J. C., 1946. The importance of colour vision to the grazing sheep. J. Agric. Sci., 39, 313.

Ulyatt M. J., 1973. The feeding value of herbages, p. 131. In Butler G. W., Bailey R. W. : Chemistry and Biochemistry of Herbages, Vol. 3, $295 \mathrm{p}$.

Weir W. C., Torell D. T., 1959. Selective grazing by sheep. $J$. Anim. Sci., 18, 641 